\title{
Living Pattern and Behavioral Risk Factors for Kala-azar: A Case-Control Study in Tertiary Care Hospital of Bangladesh
}

\author{
Afsar Ahammed ${ }^{1}$, Salahuddin Feroz ${ }^{2}$, Biswajit Bhattacharyya ${ }^{3}$, Imran Ahammed ${ }^{4}$, Shahjada Selim ${ }^{5}$, \\ Md. Abdullah Yusuf ${ }^{6}$, Md Ridwanur Rahman $^{7}$
}

\begin{abstract}
${ }^{1}$ Officer on Special Duty (OSD), Director General of Health Services, Ministry of Health \& Family Welfare, Dhaka, Bangladesh; ${ }^{2}$ Officer on Special Duty (OSD), Director General of Health Services, Ministry of Health \& Family Welfare, Dhaka, Bangladesh; ${ }^{3}$ Medical Officer, Department of Blood Transfusion. National Institute of Cardiovascular Diseases, Dhaka, Bangladesh; ${ }^{4}$ Officer on Special Duty (OSD), Director General of Health Services, Ministry of Health \& Family Welfare, Dhaka, Bangladesh; ${ }^{5}$ Assistant Professor, Department of Endocrinology, Bangabandhu Sheikh Mujib Medical University (BSMMU), Dhaka, Bangladesh; ${ }^{6}$ Assistant Professor, Department of Microbiology, National Institute of Neurosciences \& Hospital, Dhaka, Bangladesh; ${ }^{7}$ Professor, Department of Medicine, Shaheed Suhrawardy Medical College and Hospital, Dhaka, Bangladesh
\end{abstract}

[Received: 1 January 2016; Accepted: 15 July2016; Published: 1 December 2016]

\begin{abstract}
Background: There are many risk factors which can influence the causation of kala azar in Bangladeshi people. Objective: The aim of the present study is to describe the living pattern and behavioral risk factors of kala-azar among a case series of Bangladeshi patients. Method: This case control study was carried out at inpatient department of Community Based Medical College Hospital, Mymensingh from July 2010 to June 2011, for a period of 1(one) year. The study samples were clinically and parasitologically confirmed kala-azar cases. The controls were rK 39 strip test negative hospitalized cases admitted for other reason. Results: Univariate analysis showed that age, sex, marital status, average number of person living in room frequently, goat sharing in sleeping room, number of kala-azar treatment in living family members, nearest distance of known kala-azar, were risk factors. Multivariate analysis showed that Marital status (OR=6.940, $95 \% \mathrm{CI}=2.710-17.772, P=.001$ ), Number of family member living in the room were significant risk factors $(\mathrm{OR}=1.786,95 \% \mathrm{CI}=1.097-2.907, P=0.020)$ for kala-azar. Conclusion: These findings have important practical implications because they suggest that these measures, along with appropriate vector control and improved treatment facilities in rural areas, may be particularly effective in reducing the incidence of kala-azar and its transmission of infection by sand fly vectors. [Bangladesh Journal of Infectious Diseases 2016;3(2):2935]

Keywords: Kala-azar; visceral Leishmaniasis; risk factor

Correspondence: Dr. Afsar Ahammed, Officer on Special Duty (OSD), Director General of Health Services, Ministry of Health \& Family Welfare, Dhaka, Bangladesh; Email: meraz_aarm@yahoo.com; Cell no.: +8801711170680

Conflict of interest: There is no conflict of interest to any of the authors of this article.

Funding agency: The study was not funded by any authority.

Contribution to authors AA, SF, BB, IA, SS \& MRR were involved in protocol preparation, data collection and literature search up to report writing. AA was also involved in manuscript preparation and MAY for manuscript revision and in statistical analysis.

How to cite this article: Ahammed A, Feroz S, Bhattacharyya B, Ahammed I, Selim S, Yusuf MA, Rahman MR. Living Pattern and Behavioral risk factors for kala azar: a case-control study in tertiary care hospital of Bangladesh. Bangladesh J Infect Dis 2016;3(2):2935
\end{abstract}




\section{Introduction}

Visceral Leishmaniasis (VL), commonly known as Kala-azar is a chronic febrile disease caused by the protozoan parasite, Leishmania Donavan and its subspecies ${ }^{1}$. Kala-azar is characterized by chronic fever, hepato-splenomegaly, emaciation and anaemia ${ }^{2,3}$. Fever is of gradual or sudden onset. It is persistent and irregular, often with two daily peaks, with alternating periods of apyrexia and low-grade fever. Post Kala-azar Dermal Leishmaniasis (PKDL) may occur after the apparent cure of systemic disease ${ }^{4}$.It is a rural disease affecting peasants, male and young's more and if untreated, has a mortality rate of almost $100 \%{ }^{4,5,6}$. The disease agent is transmitted from the reservoir host to the susceptible host by a tiny insect usually known as sand fly $^{2,3}$ Visceral leishmaniasis occurs widely throughout the world, viz South America, South Africa, the Mediterranean countries, India, Bangladesh and China. It is now endemic in 88 countries with a total 350 million people at risk $^{4}$.Worldwide there are estimated to be approximately 500,000 cases of visceral leishmaniasis per year and many of them are associated with epidemics particularly in Indian subcontinent and Sudan ${ }^{7}$.The large number of endemic countries illustrates the global importance of the problem ${ }^{8}$.The disease is now being reported in 45 districts in Bangladesh ${ }^{9}$.The number of cases is increasing and reports from the district health authorities indicate that >15000 new cases can be expected annually ${ }^{10}$. The current prevalence is estimated as 40,000 cases. The total population at risk is more than 20 million $^{11}$. A recent study showed that the high prevalence of Kala-azar positive cases were seen in the district of Mymensingh, followed by that of Tangail, Gazipur, Dhaka, Sirajgonj and Manikganj districts of Bangladesh ${ }^{12}$. The upazilas of Trishal and Fulbaria, within the district of Mymensingh, have $60 \%$ of all cases in the country according to the Ministry of Health ${ }^{9}$. An entomological survey shows density of sand flies both vector and non-vector species were significantly higher in endemic areas than non-endemic areas ${ }^{13}$.Now a days, the disease shows wide spread geographical distribution and is being reported from previously non-endemic areas. Economic and demographic circumstances that contribute to increased prevalence include: new agroindustrial project, large-scale migration of populations, unplanned urbanization, and man-made environmental changes ${ }^{14}$.In common with its neighbors' India and Nepal, VL in Bangladesh is anthroponotic. Living in proximity to a Kala azar case is the strongest risk factor for the disease ${ }^{15}$. Bed net use, sleeping on a cot and indoor residual spraying are generally associated with decreased risk. Poor micronutrient status increase the risk of progression to Kala $\operatorname{azar}^{16}$.

Elimination of infectious disease is an ultimate goal to the public health system and therefore, the efforts have recently gained momentum from various organizations such as WHO/TDR, WHO-SEARO, World Bank, UNICEF, Bill and Melinda Gates Foundation, Drugs for Neglected Diseases initiative (DNDi) and the Government of Bangladesh. Despite the implementation of various control programs the occurrence of Kala-azar in several districts has been constant feature. This is partly due to inadequate information about the various risk factor associated with the disease. Very little is known about the influence of socioeconomic, living standard and behavioral factors on Kala-azar in disease endemic of Bangladesh. Research activities concerning Kala-azar have primarily focused on the characteristics of the parasite and the host. Therefore this case control study will help to determine whether socioeconomic, living pattern and behavioral characteristics and lack of proper vector control are risk factor for occurrence of Kala-azar.

\section{Methodology}

The study was carried out at the Community Based Medical College Hospital, in Mymensingh district from July 2010 to June 2011. The study is designed as a case control study. Patients admitting the inpatient department of Community Based Medical College Hospital, Mymensingh during the study period. The cases were were rK 39 strip test positive and parasitologically confirmed cases of Visceral Leishmaniasis and the controls were rK 39 strip test negative hospitalized cases admitted for other reason. Since no prior quantitative information on various risk factors was available, estimated visceral leishmaniasis prevalence in Indian sub-continent (Bangladesh, India and Nepal) was found 21 cases per 10,000 sample population ${ }^{17}$. As the available number of cases in the setup is not many, over the study duration we could recruit 60 cases and 120 controls for the present case series. The case control ratio was 1:2. The Study subjects were recruited from the cases enrolled for a Randomized Controlled Trial at the study place. They were primary cases i.e., has never been diagnosed or treated for Kala azar in past. A separate consent process was followed for the present 
case-control study. As part of the Randomized Control Trial the cases were rK39 strip test positive and already confirmed by splenic aspiration positive for LD bodies. The controls were approached for written informed consent process to participate in the study and negative rK39 strip test. Inclusion criteriaincludes male or female of all ages with rk39 positive at baseline assessments and history of fever, for at least 2 weeks with Anaemia $(5<\mathrm{Hb}<10 \mathrm{~g} / \mathrm{dl})$ and/or Loss of weight and/or Splenomegaly. Exclusion criteria were known Hepatitis or known HIV positive, patients who present with Post Kala-azar Dermal Leishmaniasis, Clinical symptoms of chronic underlying disease such as severe cardiac, renal or hepatic impairment, patient with Positive HRP2/pLDH Combo test for Malaria and patient unwilling to participate. Interview schedule and check list were uses as research tool. The pre-designed semi-structured questionnaires were used for both the cases and controls. A face to face interview with the cases and controls and in case of cases unable to respond for the illness, spouse or the closest attendant were interviewed for filling out the questionnaire. The variables included to record/measure were age, sex, BMI, income, marital status, average number of persons living in room frequently, living in floor frequently, using mosquito net, animal sharing in sleeping room or nearer to sleeping room, number of kala azar treatment in family, nearest distance of known kala azar case, food consumption habit. Data were entered into a computer and data file was constructed. Forms were decoded and scrutinized for accuracy and consistency. Data were analyzed using Epi-Info version 6.2 (Centers for Disease Control and Prevention, Atlanta, GA) and validated by logical and range checks with SPSS version 16.0. For each of the study factors, risk was estimated by calculating the odds ratio (OR) as an approximation of the relative risk with 95\% confidence intervals (CIs) using EpiInfo version 6.2. The significance of the OR was analyzed with the Mantel-Haenszel chi-square test. On the basis of the OR in univariate analysis, variables that were statistically significant were included in the multiple logistic regression model. Stepwise multiple logistic regression was then applied for the identification of significant risk factors using SPSS version 16.0.Approval from the Bangladesh Medical Research Council (BMRC) was taken prior to commencement of the study. The study was reviewed and approved by the Scientific Advisory Committee (SAC) and Institutional Ethical Committee. Informed consent was obtained from all cases and controls before including them into study.I

\section{Results}

The distribution of the cases and controls by age, sex, family size, and Living Standard Index is shown in Table 1. Differences in the proportion in each age group $(P=0.001)$, sex $(P=0.010)$, average family size $(P=0.507)$, and LSI $(P=0.293)$, religion $(\mathrm{P}=0.839)$ of cases and controls were not statistically significant, except age. The LSI indicated that most of the cases and controls belonged to lower middle class (>4000tk) socioeconomic strata of the community. This result indicated that primary socioeconomic and demographic characteristics were similar in both cases and controls except the age group of cases are younger than those of controls.

Table: 1. Socio demographic characteristics of cases and controls

\begin{tabular}{llll}
\hline Factors & $\begin{array}{l}\text { Cases } \\
(\mathbf{n = 6 0})\end{array}$ & $\begin{array}{l}\text { Controls } \\
(\mathbf{n = 1 2 0})\end{array}$ & $\boldsymbol{P}$ \\
\hline Age (in year) & & & \\
$\leq 10$ & $19(31.7 \%)$ & $7(5.8 \%)$ & 0.001 \\
$11-20$ & $16(26.7 \%)$ & $11(9.2 \%)$ & \\
$21-30$ & $9(15.0 \%)$ & $34(28.3 \%)$ & \\
$31-40$ & $9(15.0 \%)$ & $24(20.0 \%)$ & \\
$41-50$ & $7(11.7 \%)$ & $20(16.7 \%)$ & \\
$\geq 50$ & $0(0 \%)$ & $24(20.0 \%)$ & \\
Mean $\pm \mathrm{SD}$ & $21.52 \pm 14.14$ & $36.30 \pm 15.31$ & \\
Sex & & & \\
Male & $43(71.7 \%)$ & $62(51.7 \%)$ & 0.010 \\
Female & $17(28.3 \%)$ & $58(48.3 \%)$ &
\end{tabular}

\section{Religion}

Islam

$56(93.3 \%)$

$111(92.5 \%)$

0.839

Hindu

$4(6.7 \%) \quad 9(7.5 \%)$

Marital

Status

$\begin{array}{llll}\text { Unmarried } & 35(58.3 \%) & 20(16.7 \%) & .001\end{array}$

Married 25(41.7\%) 100(83.3\%)

\section{Living Standard Index}

$\begin{array}{lll}\leq 2000 & 1(1.7 \%) & 0(.0 \%) \\ 2001-4000 & 17(28.3 \%) & 29(24.2 \%) \\ \geq 4000 & 42(70.0 \%) & 91(75.8 \%)\end{array}$




\begin{tabular}{lll}
\hline Factors & $\begin{array}{l}\text { Cases } \\
(\mathbf{n = 6 0})\end{array}$ & $\begin{array}{l}\text { Controls } \\
(\mathbf{n = 1 2 0})\end{array}$ \\
\hline Occupation & & \\
Student & $25(41.7 \%)$ & $17(14.2 \%)$ \\
Farmer & $11(18.3 \%)$ & $19(15.8 \%)$ \\
Housewife & $6(10.0 \%)$ & $46(38.3 \%)$ \\
Day labourer & $4(6.7 \%)$ & $7(5.8 \%)$ \\
Businessman & $4(6.7 \%)$ & $12(10.0 \%)$ \\
Dependent & $3(5.0 \%)$ & $1(0.8 \%)$
\end{tabular}

\begin{tabular}{llll}
\hline Factors & $\begin{array}{l}\text { Cases } \\
(\mathbf{n = 6 0 )}\end{array}$ & $\begin{array}{l}\text { Controls } \\
(\mathbf{n = 1 2 0})\end{array}$ & $\boldsymbol{P}$ \\
\hline Service holder & $7(11.7 \%)$ & $18(15.0 \%)$ &
\end{tabular}

Number of family members (Mean \pm SD.)

Number of

family $\quad 5.48 \pm 1.70 \quad 5.67 \pm 1.82 \quad 0.507$

members

Table: 2. Living and Behavioral Factors

\begin{tabular}{|c|c|c|c|}
\hline Factors & Cases $(n=60)$ & Controls $(n=120)$ & $\boldsymbol{P}$ \\
\hline \multicolumn{4}{|l|}{ Number of family members (Mean \pm SD) } \\
\hline Number of family members & $5.48 \pm 1.70$ & $5.67 \pm 1.82$ & 0.507 \\
\hline \multicolumn{4}{|c|}{ Average number of person living in room frequently (Mean \pm SD) } \\
\hline $\begin{array}{l}\text { average number of person living in } \\
\text { room frequently }\end{array}$ & $3.00 \pm 1.04$ & $2.48 \pm 0.82$ & 0.001 \\
\hline \multicolumn{4}{|l|}{ Living in the floor frequently } \\
\hline Living in the floor frequently & $3(5.0 \%)$ & $1(0.8 \%)$ & 0.074 \\
\hline \multicolumn{4}{|l|}{ Using mosquito net } \\
\hline Yes & $38(63.3 \%)$ & $79(65.8 \%)$ & 0.740 \\
\hline No & $22(36.7 \%)$ & $41(34.2 \%)$ & \\
\hline \multicolumn{4}{|c|}{ Animal share in sleeping room or kept next to sleeping room } \\
\hline Goat & $11(18.3 \%)$ & $9(7.5 \%)$ & 0.029 \\
\hline Chickens & $19(31.7 \%)$ & $34(28.3 \%)$ & 0.644 \\
\hline Cow & $11(18.3 \%)$ & $33(27.5 \%)$ & 0.177 \\
\hline
\end{tabular}

Number of $\mathrm{VL} / \mathrm{Kala}$ azar treatment in living family members (Mean \pm SD)

Number of VL/Kala azar treatment in
living family members
$0.83 \pm 1.12$
$0.08 \pm 0.35$
0.001

Nearest distance of a known case of kala azar

Within household

Others

Food consumption

Eat meat (at least twice a week)

Eat fish (at least twice/thrice a week)

Eat fruits (at least twice/thrice a week)
$28(46.7 \%)$

$32(53.3 \%)$

$7(5.8 \%)$

$113(94.2 \%)$

$10(16.7 \%)$

$57(95.0 \%)$

$20(33.3 \%)$
$12(10.0 \%) \quad 0.198$

$119(99.2 \%) \quad 0.109$

$26(21.7 \%) \quad 0.091$
The distribution of the cases and controls by age, sex, family size, and Income is shown in Table 1.
Differences in the proportion in each average family size $(P=0.507)$, and Income $(P=0.293)$, Religion 
$(p=0.839)$ longest residence in kala azar area $(p=0.732)$, number of family members $(p=0.507)$ of cases and controls were not statistically significant. The Income indicated that most of the cases and controls belonged to low socioeconomic strata of the community. This result indicated that primary socioeconomic and demographic characteristics were similar in both cases and controls. The unadjusted
ORs of risk factors for individual and family characteristics is shown in Tables 2. The results showed that age, gender, marital status, BMI, average number of person living in the room, kala azar treatment in living family members, nearest distance of a known case of kala azar were significant risk factors by univariate analysis.

Table: 3. Risk factors for Kala-azar by multivariate analysis using a logistic regression model

\begin{tabular}{llllll}
\hline Variable & $\begin{array}{l}\text { Regression } \\
\text { coefficient }\end{array}$ & SE & $\begin{array}{l}\text { Odds } \\
\text { ratio }\end{array}$ & 95\% CI & p value \\
\hline Marital Status & 1.937 & .480 & 6.940 & $2.710-17.772$ & .001 \\
Number of person in living room & .580 & .248 & 1.786 & $1.097-2.907$ & .020 \\
Animal share in sleeping room & .771 & .718 & 2.163 & $.529-8.836$ & .283 \\
Number of treated kala azar in living family & 1.027 & .952 & 2.794 & $.432-18.053$ & .281 \\
members & 1.320 & 1.311 & 3.743 & $.286-48.910$ & .314 \\
Nearest distance of knowing kala azar & & & & & \\
\hline
\end{tabular}

$\mathrm{CI}=$ Confidence Interval

The results of multiple logistic regression analysis are shown in Table 3. The significant risk factors on the basis of adjusted ORs for kala-azar in multivariate analysis were identified. Some factors, which were significant in univariate analysis, were not significant risk factors in multivariate analysis. The adjusted ORs and 95\% CIs for each significant variable are shown in Table 2. Marital status shows that unmarried significantly associated with kalaazar $(\mathrm{OR}=6.940,95 \% \mathrm{CI}=2.710-17.772 P=$ $0.001)$.Number of family member living in the room was also a risk factor $(\mathrm{OR}=1.786,95 \% \mathrm{CI}=$ 1.097-2.907, $P=0.020)$. The number of kala azar treated family member within the house $(\mathrm{p}=.001)$, goat share in sleeping room or kept next to sleeping room $(\mathrm{p}=.029)$ associated with significant risk factor in univariate analysis but not significant association in multivariate analysis. Number of person in living room shows significant risk association with kala azar in multivariate analysis $(\mathrm{OR}=1.786,95 \% \mathrm{CI}=1.097-2.907, P=0.020)$.

\section{Discussion}

This study is probably the first attempt to assess the association of various socioeconomic, living pattern and behavioral factors with occurrence of kala-azar using a case-control design approach. Distribution of family income by groups showed that $70 \%$ of the cases had monthly income >4000tk and was not significant in univariate analysis. It was previously reported that most kala-azar patients had incomes less than $\$ 1$ per day $(80 \text { taka })^{18}$. Such poverty may not be a risk factor for kala-azar, but it can lead to malnutrition (BMI), poor housing conditions, lack of preventive measures in the form of sanitation and bed nets, and illiteracy. Thus, poverty could be a major determinant for continued transmission of Kala-azar .In this study age and sex distribution has no significant risk association with kala azar in multivariate analysis but significantly influenced kala azar association in univariate analysis. Much younger age group and a higher male dominance in univariate analysis reflects that increase activities may lead them to exposed different environment that might have risk association with kala azar.Though this is unmatched case-control study and no significant risk association between kala azar and marital status has been reported but in this study it was found that unmarried person suffered significantly more in comparison to married person. Although animal share in sleeping room was not found significant risk factors in multivariate analysis in this study but interestingly presence of goat in sleeping room or next to sleeping room was associated with kala azar. Presence of domestic 
animals such as dogs, pigs, rodents and other kala azar cases has been associated with kala azar which was in contrast with our study ${ }^{19}$.

It was observed that the risk of kala-azar was higher among cases with a history of kala-azar among the family members in the past year compared with those cases with no history of kala-azar among family members. The presence of kala-azar cases in the family might aid the transmission of this disease in the presence of sand fly vectors and other conditions favorable for completion of transmission cycle within the house which is indicative of the short flight range of sandflies ${ }^{20}$.Though multivariate analysis show no significant risk association but presence of kala azar treated person in affected individual significant risk association in univariate analysis. In this study a higher number of person living in one room was found significant risk association with kala azar, greater density in terms of household members increases the exposure of household members to sandflies ${ }^{21}$.

Thus, the socioeconomic characteristics identified as risk factors of VL in this study could help strengthen existing control strategies. Better housing and improved living conditions in diseaseareas could reduce the transmission of the disease by eliminating conditions suitable for breeding of sand flies inside the houses. Proper implementation of existing health awareness programs could help people in rural areas modify their behavioral patterns. The main emphasis on targeting intervention would be enhancing community awareness in areas susceptible to kala-azar through dissemination of appropriate information. Women groups and local non-governmental organizations should also be actively involved in awareness programs. These findings have important practical implications because they suggest that these measures, along with appropriate vector control and improved treatment facilities in rural areas, may be particularly effective in reducing the incidence of kala-azar and its transmission of infection by sand fly vectors.

This is a hospital based unmatched case-control study. The hospital was in the city. So the study could not collect the representative samples. Most of the patients were from the urban or semi urban areas. So the rural patients were lacking. We did not have enough resource and scopes.

\section{Conclusion}

This small case control study reveals Marital status, Number of family member living in the room were the major risk factors for development of kala azar in Mymensingh region of Bangladesh. Further studies should be carried out with larger sample size and covering other Kala azar regions of the country to improve the precision of quantification of risk factors in a country perspective.

\section{References}

1. WHO Technical Report Series. No. 701, 1984. The Leishmaniasis, report of expert committee

2. Marinkelle CJ. The control of Leishmaniasis. Bulletin of the World Health Organization.1980.58:807

3. Chatterjee KD. Parasitology (Protozoology and Helminthology). $12^{\text {th }}$ edition, 1980.

4. Park K. Park's Textbook of Preventive and Social Medicine. 16th edn. 2000, M/S Banarsidas Bhanot Publishers, Jabalpur, India: 231-233

5. Chowdhury MS, Ahmed MTU, Hussain AMZ, et al. Visceral leishmaniasis its control.1st ed. Dhaka: IEDCR; 1993; 4 - 28

6. Rashid KM, Khabir M, Haider S. The textbook of Com. Med. \& Public Health. 3nd ed. Dhaka: RKH publishers; 1999: 317 - 322

7. TK. Jha, Shayam Sundar, Thakur, Bachmann Peter, Juntra Kabwang, Chris Fisher, Andres Voss et.al .Multefosine, an oral agent for the treatment of Indian Visceral Leishmaniasis. The New England Journal of Medicine. 1999; 341 (24):17995-99

8. WHO (1996) World Health Report 1996.Report of Director -General WHO.

9. Hope for Kala azar sufferers in Bangladesh, Medicins Sans Frontieres, doctors without borders, 19 june 2010

10. Laboratory diagnosis of kala-azar (Visceral leishmaniasis) with the Direct Agglutination Test (DAT) - Atraining module for laboratory Technician, Published by IEDCR and M\&PDC, DGHS, 1996

11. Kala-azar (Visceral Leishmaniasis) Diagnostic, Treatment and recording charts- A training module for medical officers' .Published by M \& PDC and IEDCR, 1995

12. Begum N, Masum MA, Mamoon AB, Begum A. Visceral leishmaniasis among the suspected febrile patients. Bangladesh Journal of Medicine Jan, 2002; 13: 21 - 25

13. Alam MS, Wagatsuma Y, Mondal D, Khanum H, Haque R. Relationship between sand fly fauna and Kala azar endemicity in Bangladesh. Journal Acta Tropica 2009; 112:23-25

14. English report on the world health situation, Implementation of the global strategy for Health for All by the year 2000.Second evaluation, Vol.1, Global review.1993

15. Cary Bern \& Rajib Chowdhury.The epidemiology of visceral leishmaniasis in Bangladesh: prospects for improved control. Indian J Med. Res123, March 2006; pp. 275-288 
16. Bern C, Courtenay O, Alvar J. Of cattle, sand flies and men: a systemic review of risk factor analysis for South Asian visceral leishmaniasis implication for elimination, Journal PLoS Neglected Tropical Diseases, February 2010; ISSN: 1935-2727

17. National guideline on Kala azar Elimination Program in Bangladesh, 2010

18. Thakur CP. Socio-economies of visceral leishmaniasis in Bihar (India). Trans R Soc Trop Med Hyg 2000; 94: 156157
19. Barnett PG, Sing SP, Bern C, Hightower AW, Sundar S. Virgin Soil: the spread of visceral leishmaniasis into Uttar Pradesh, India.AM J Trop Med Hyg 2005; 73:720-725

20. Killick-Kendrick R. The biology and control of phlebotomine sand flies. Clin Dermatol 1999; 17: 279-289

21. Reithinger R, Mohsen M, Leslie T. Risk factors for anthroponotic cutaneous leishmaniasis at the household level in Kabul, Afghanistan. PLoS Negl Trop Dis 2010; $4: 639$ 